\title{
EVALUACIÓN DE LA CALIDAD DE UN SERVICIO DE VOLUNTARIADO EN ONCOLOGÍA: UN ANÁLISIS IMPORTANCIA-VALORACIÓN (IPA)
}

\author{
QUALITY ASSESSMENT OF A VOLUNTEER SERVICE IN ONCOLOGY: AN \\ IMPORTANCE PERFORMANCE ANLYSIS (IPA)
}

\author{
Luis Gil Calzada', Ainhoa Carrasco Estévez', Víctor Manuel Muñoz Garzón² \\ ' Asociación Española Contra el Cáncer. \\ 2 Servicio de Oncología Radioterápica del Hospital do Meixoeiro de Vigo.
}

Resumen

Objetivo: Usando el Análisis de Importancia-Valoración (IPA), este artículo examina la importancia y valoración percibidas por pacientes oncológicos y sus acompañantes de un programa de humanización realizado por voluntarios en un servicio de Radioterapia de un hospital general en España.

Método: Los autores identifican una lista de siete ítems que surgen de la revisión de literatura en Marketing Sanitario; cada ítem fue puntuado con una escala Likert de 5 puntos.

Resultados: Los resultados se obtuvieron de las respuestas a 148 entrevistas. El gráfico fue corregido con las recomendaciones sugeridas en la literatura y en el caso de los pacientes, muestra tres factores que caen en el cuadrante de "Mantener el buen trabajo", cuatro atributos caen dentro del cuadrante de "Baja Prioridad" y ningún atributo se observa en los cuadrantes de "Posible dilapidación de recursos" y "Concentrarse aquí". Se encuentran diferencias entre medias de la muestra de pacientes $(n=68)$ y de familiares acompañantes $(n=80)$ en los atributos de catering $(t=-$ 2,38; g.l.=146; $p<0,05)$, y en el de información $(t=2,16 ; g . I .=146 ; p<0,05)$.

Conclusiones: Los resultados parecen útiles para identificar áreas de interés para los gestores de los servicios de salud para desarrollar programas de humanización de servicios basa-

\section{Abstract}

Objective: Using an Importance-Performance Analysis (IPA), this paper examined a Radiotherapy and Oncological Patient and Non-patient perceived importance and performance of ten Humanization Volunteer Program selection factors in the General Hospital in Spain.

Methods: The authors identified a list of seven items from the Health-marketing literature reviews, and each item was rated using a 5-point Likert scale. Responses were obtained of 148 usable interviews.

Results: The importance-performance patient grid was corrected with literature recommendations and, in the patient sample, shows three items fall in the "Keep up the good work" quadrant, four items fall into the "Low priority" quadrant, zero items fall into the "Possible overkill" quadrant, and no items fall in the "Concentrate here" quadrant. Nonpatient $(n=80)$ factors means shows statistical differences with patient $(n=68)$ means in catering ( $t=-2.38$; $d f .=146 ; p<0.05)$, and information $(t=2.16 ; d f 146 ; p<0,05)$.

Conclusions: The results are useful in identifying areas for strategic focus to help Health Services managers develop humanization programs with volunteer workers and different program users. Implication to volunteer programs managers and researchers were discussed.

Correspondencia:

Dr. Luis Gil Calzada

C/ Doutor Marañón, 11-4%;

E-mail: lvisgilcalzada@msn.com 
dos en el trabajo de voluntarios y dirigidos a diferentes tipos de usuarios. Las implicaciones para los gerentes de programas de voluntariado e investigadores son discutidas.

Palabras clave: análisis importancia-valoración, voluntarios, radioterapia, sala de espera.
Keywords: Importance-performance analysis, volunteer, radiotherapy, waiting room.

\section{INTRODUCCIÓN}

Los servicios prestados por voluntariado en el ámbito del tratamiento de la enfermedad oncológica son cada día más importantes y numerosos. El impacto dentro de los cuidados paliativos del trabajo de voluntariado se expresa en algunas revisiones de una literatura cada vez más extensa $^{(1,2)}$. Los resultados de esta literatura muestran que los servicios llevados a cabo por voluntarios logran resultados significativos según los últimos meta-análisis ${ }^{(1)}$. No obstante, la mayoría de resultados hacen referencia al campo de cuidados paliativos dentro de programas basados en la evidencia en el entorno de hospitales especializados en cuidados paliativos ${ }^{(3-7)}$. Sin embargo, el servicio de voluntariado también se utiliza en otros entornos como programas para la prevención ${ }^{(1,8)}$, el apoyo durante el tratamiento ${ }^{(1,9)}$ y cuidados y apoyo durante el seguimiento en supervivientes ${ }^{(1,2,8)}$. Todos estos servicios hacen que cada vez sea más complejo el manejo del voluntariado para los gerentes que los organizan.

Actualmente, los trabajos de investigación dirigidos a la mejora de los recursos de los gerentes de servicios basados en el voluntariado se han centrado en aclarar los perfiles básicos de los voluntarios y, dentro de estos, las variables que los llevan a permanecer más tiempo en sus puestos ${ }^{(3-7)}$. Se han descrito las diferentes motivaciones que llevan a una persona a hacerse voluntario dentro de los cuidados paliativos, siendo las más comúnmente encontradas las motivaciones altruistas, las de respon- sabilidad cívica, las de auto-promoción y las de ocio, hay datos que relacionan, además, la motivación altruista con una mayor permanencia como voluntario a lo largo del tiempo ${ }^{(4,7)}$. Los resultados sugieren que los motivos altruistas tuvieron mayor influencia en la mayoría de voluntarios. También aparecen diferencias culturales en las motivaciones para hacerse voluntario dentro de estos campos $^{(4-7)}$. Por último, se han estudiado los perfiles de personalidad asociados a desarrollar la labor de voluntariado, obteniendo que los voluntarios que se dedican a cuidados paliativos en el hospital especializado puntúan más bajo en la dimensión de Neuroticismo, mientras que puntúan más alto en Amabilidad y Responsabilidad que la población general(5). Todos estos resultados pueden ayudar a mejorar los servicios de voluntariado tanto en la selección como en el cuidado de los propios voluntarios a lo largo de su servicio, sin embargo, no cubren las necesidades de evaluación de calidad y seguimiento de los programas basados en voluntariado.

Enmarcados en los servicios de voluntariado en el contexto hospitalario, actualmente se realizan servicios de apoyo y acompañamiento durante la espera en las salas dedicadas a dicho fin para consultas o tratamientos de radioterapia. Los profesionales sanitarios intentan tener en cuenta el impacto de la espera como estresor, que debe ser minimizado lo máximo posible, y para ello utilizan en esa espera los servicios de voluntariado junto con la oferta de un catering que pueda reducir el impacto del distrés anticipatorio(10,11). En 
este contexto existen numerosos fenómenos que sufren los pacientes de estos servicios; en caso de acudir solos, la espera es acompañada de sentimientos de angustia, aburrimiento y en casos extremos, de ansiedad $^{(10,11)}$. Los equipos de programas de voluntariado ofrecen acompañamiento y apoyo, junto al catering, tanto a pacientes como a familiares/acompañantes, para poder minimizar todo lo posible el impacto del estrés de la espera y lograr la percepción de humanización del contexto hospitalario. Estos programas necesitan ser evaluados para saber si están siendo satisfactorios entre los usuarios a los que van dirigidos. Asimismo, los gerentes de este tipo de servicios necesitan recursos para la medición fiable y válida de la calidad de este tipo de programas.

Con el objeto de mejorar el conocimiento general, así como el de la gestión y recursos dirigidos a la evaluación de la calidad de los servicios prestados por voluntarios, se ha realizado este estudio prospectivo. Durante años ha habido muchos estudios que proponen diferentes técnicas para el estudio de la calidad de servicios de salud y hospitalarios ${ }^{(12-18)}$. Ha demostrado una especial utilidad la técnica Ilamada Análisis de la Importancia-Valoración, propuesta por primera vez por Martilla y James en el año $1977^{(19)}$. Las ventajas fundamentales de esta técnica son la facilidad de aplicación, la facilidad de interpretación de los resultados y el estar dirigida a aspectos prácticos de la gerencia de servicios $^{(19-21)}$. Otra ventaja es su utilización previa para la medición de la calidad en el sector de servicios de la salud y también en el Hospital. Además, hay varios estudios en muestras españolas y esto último es una ventaja ya que se ha encontrado que la técnica es sensible a diferencias culturales en otros entornos ${ }^{(22,23)}$.

Esta técnica se basa en las teorías de expectativa-valor y en las teorías de la discrepancia de las expectativas ${ }^{(22-27)}$. Su fun- cionamiento tiene como hitos principales la selección de atributos que definen al servicio a evaluar, la medición de la importancia de cada atributo en una muestra de usuarios del servicio, la medición de la valoración de cada atributo en la misma muestra anterior y la representación gráfica en dos ejes de los datos encontrados, que permite interpretar los resultados en 4 decisiones gerenciales: "concentrarse aquí, mantener el buen trabajo, baja prioridad y posible dilapidación de recursos". En cada hito se han detectado diversos problemas metodológicos desde la creación de la técnica, siendo las más importantes críticas la manera de medir la importancia y por tanto de conceptualizarla, el posicionamiento de los ejes según los sesgos de respuesta de los usuarios para reducir el campo de análisis y la medición y representación de las discrepancias entre importancia y valoración como medida complementaria de la calidad del servicio ${ }^{(20,22,23)}$. Todas estas áreas deben modificarse respecto a la propuesta inicial de Martilla y James ${ }^{(19)}$, para poder corregir errores y sesgos que la técnica original incluía y para ello hay diferentes propuestas como el reposicionamiento de los ejes, la medición de la importancia con puntuaciones directas y la inclusión de la discrepancia de las medidas mediante la inclusión de la diagonal en el gráfico IPA (Importance Performance Analysis) ${ }^{(20,22,23)}$.

Este estudio tiene varios objetivos: primero nos proponemos probar la metodología IPA en la evaluación de calidad de servicios de voluntariado dentro del contexto hospitalario para poder proponerla como una herramienta eficaz y eficiente para los gerentes de estos programas. Segundo, queremos medir la calidad del servicio, bajo la hipótesis de que éste es un servicio percibido con una alta satisfacción, con altas medidas de importancia y valoración, y finalmente con una medida diferencial de importancia y valoración positiva en la 
mayoría de los atributos. Tercero, deseamos observar si se confirma la hipótesis de que los familiares acompañantes y pacientes muestran diferencias en la percepción de calidad del servicio, ya que son poblaciones con necesidades diferentes al exponerse a la situación de espera en la sala del Hospital.

\section{MÉTODO}

Como se especifica en el trabajo original de Martilla y James de 1977, la técnica IPA tiene una fase previa en la que hay que definir los atributos que conforman el producto o servicio, que luego habrá que evaluar por su importancia y valoración con un diseño de encuesta a los usua$\operatorname{rios}^{(19)}$. En este trabajo esta fase previa se realiza tras una revisión de la literatura especializada en la técnica y el sector concreto desde 1960 a 2013, utilizando como palabras clave: IPA, voluntariado, calidad del servicio. Este procedimiento es uno de los propuestos en el origen y ha sido ampliamente utilizado. A posteriori se utilizó el criterio experto de 2 médicos especialistas en Oncología Radioterápica, 3 psicólogos, 1 trabajadora social, 2 enfermeras y la Directora Gerente del servicio, que decidieron cuales serían los atributos finales para evaluar el programa. Tras este procedimiento se establecieron los siguientes atributos: escucha a pacientes y familiares, proporcionar compañía, facilitar entretenimiento, ofrecer información útil, proporcionar apoyo emocional, humanización y catering. Finalmente se establece que se evaluará una percepción general del servicio.

La segunda fase de la técnica IPA consiste en la evaluación de estos atributos en una muestra de usuarios del servicio según un muestreo intencional en horario de mañana y tarde, siendo la entrevista anónima con consentimiento verbal. La duración aproximada fue de entre 2 y
7 minutos, siendo los encuestadores voluntarios de Asociación Española Contra el Cáncer formados específicamente para esta tarea concreta.

La muestra se compone de 148 usuarios y está estratificada en 68 pacientes oncológicos y 80 familiares/acompañantes que fueron encuestados mediante una entrevista cara a cara cuando esperaban para consulta y/o tratamiento en la sala de espera del Servicio de Oncología Radioterápica del Hospital Meixoeiro de Vigo. La selección de los participantes se realizó por orden de llegada de los usuarios a las salas de espera durante un periodo de un mes entre abril y mayo de 2013, descartando usuarios que no pudieran responder a la entrevista por razones físicas o mentales evaluadas por los entrevistadores. Los criterios de inclusión en el estudio fueron: 1 . Ser paciente o acompañante/ familiar del paciente que asiste a servicios de Oncología y tener contacto previo con el programa de voluntariado. 2. Tener capacidad para responder a las preguntas y ser mayor de edad. 3. Aceptar realizar la entrevista anónima. Los criterios de exclusión del estudio fueron: 1 . No ser paciente o acompañante de paciente de un servicio de Oncología o no haber tenido contacto con el servicio de voluntariado. 2. No tener capacidad para responder a la entrevista o no ser mayor de edad. 3. No aceptar la realización de la entrevista. De las 162 entrevistas iniciadas se consiguieron realizar un total de 148 entrevistas válidas (91,4\% de entrevistas válidas). Ver tabla 1 de datos sociodemográficos.

La entrevista se diseñó de manera que los usuarios respondan con una escala tipo Likert de 5 valores. De los 8 atributos seleccionados se realizaron dos bloques de preguntas de modo que en uno se evalúa la importancia percibida de cada atributo y en el otro se valora cada atributo. Se separaron los dos bloques con la realización de otras preguntas generales entre un bloque y el 
otro, lo que permite reducir sesgos por la proximidad de las preguntas, tal como se recomienda desde los orígenes de la técnica ${ }^{(19)}$. Véase la entrevista en el anexo 1.

Tras la fase de trabajo de campo, se analizan los datos a través de la gráfica IPA. Los diferentes trabajos sobre esta técnica recomiendan que se utilice una serie de cambios que mejoran la interpretación de los datos. La primera mejora es mover los ejes: en vez de utilizar el punto medio de la escala, se utiliza la media de cada dimensión (importancia, valoración), de modo que encontramos que la gráfica se centra en la zona de mayor importancia y valoración. Tras mover los ejes, debido al sesgo que hace que las puntuaciones tiendan a ser más altas que el punto medio, graficamos a partir del punto de la escala que tenga valores, reduciendo el campo de observación lo máximo posible. Finalmente se realiza una representación de la diagonal que permite la interpretación visual de los atributos que se perciben con margen de mejora. Con esta diagonal, los atributos que están por encima de la línea representan aquellos cuya valoración es menor que la importancia percibida. Esta última mejora de la gráfica permite una visualización rápida de la discrepancia entre la expectativa y el valor del atributo concreto. En este trabajo hemos utilizado las prescripciones recomendadas en trabajos previos, con una especial atención a las recomendaciones realizadas por el trabajo de Abalo Piñeiro et al. ${ }^{(22,23)}$.

\section{RESULTADOS}

La distribución por sexo resultó ser de $51(34,5 \%)$ hombres y $97(65,5 \%)$ mujeres. El $50 \%$ de la muestra residen en la zona urbana de Vigo y Pontevedra. El 38,3\% de diagnósticos correspondían a cáncer de mama, el 12,8\% de los usuarios acudían por causa de un diagnóstico de cáncer de pulmón, un 10,6\% correspondían a cáncer de próstata y un $6,4 \%$ de cáncer de colon, situándose el resto de diagnósticos por debajo del 5\%. La media de edad es de 53,18 \pm DT=14,14 años. Ver tabla 1 de datos sociodemográficos.

Tabla 1. Datos sociodemográficos

\begin{tabular}{|l|l|l|}
\hline Género & 51 & $34,5 \%$ \\
\hline Hombres & 97 & $65,5 \%$ \\
\hline Mujeres & 47 & $31,8 \%$ \\
\hline Edad & 48 & $32,4 \%$ \\
\hline De 18 a 48 años & 53 & $35,8 \%$ \\
\hline De 49 a 59 años & 36 & $38,3 \%$ \\
\hline De 60 a 80 años & 12 & $12,8 \%$ \\
\hline Tipo de cáncer & 10 & $10,6 \%$ \\
\hline Mama & 6 & $6,4 \%$ \\
\hline Pulmón & 30 & $31,9 \%$ \\
\hline Próstata & 54 & \\
\hline Colon & 74 & $50 \%$ \\
\hline Otros & 74 & $50 \%$ \\
\hline No diagnosticado & 74 \\
\hline Lugar de residencia &
\end{tabular}


Tabla 2. Medias y diferencias de la Importancia y la valoración del servicio

\begin{tabular}{|l|c|c|c|c|}
\hline & \multirow{2}{*}{$n$} & VALORACIÓN & IMPORTANCIA & DIF. \\
\cline { 3 - 5 } & & \multicolumn{3}{|c|}{ Media } \\
\hline PROPORCIONAR COMPAÑIA & 148 & 4,51 & 4,43 & 0,08 \\
\hline ESCUCHA A PACIENTES Y FAMILIARES & 148 & 4,52 & 4,53 & $-0,01$ \\
\hline PROPORCIONA APOYO EMOCIONAL & 148 & 4,45 & 4,50 & $-0,05$ \\
\hline FACILITA ENTRETENIMIENTO & 148 & 4,58 & 4,54 & 0,04 \\
\hline HUMANIZACIÓN & 148 & 4,76 & 4,73 & 0,03 \\
\hline OFRECE INFORMACIÓN ÚTIL & 148 & 4,16 & 4,23 & $-0,07$ \\
\hline CATERING & 148 & 4,66 & 4,56 & 0,1 \\
\hline PERCEPCIÓN GENERAL DEL SERVICIO & 148 & 4,78 & 4,70 & 0,08 \\
\hline
\end{tabular}

Gráfico 1. IPA de los usuarios ( $n=148$ )

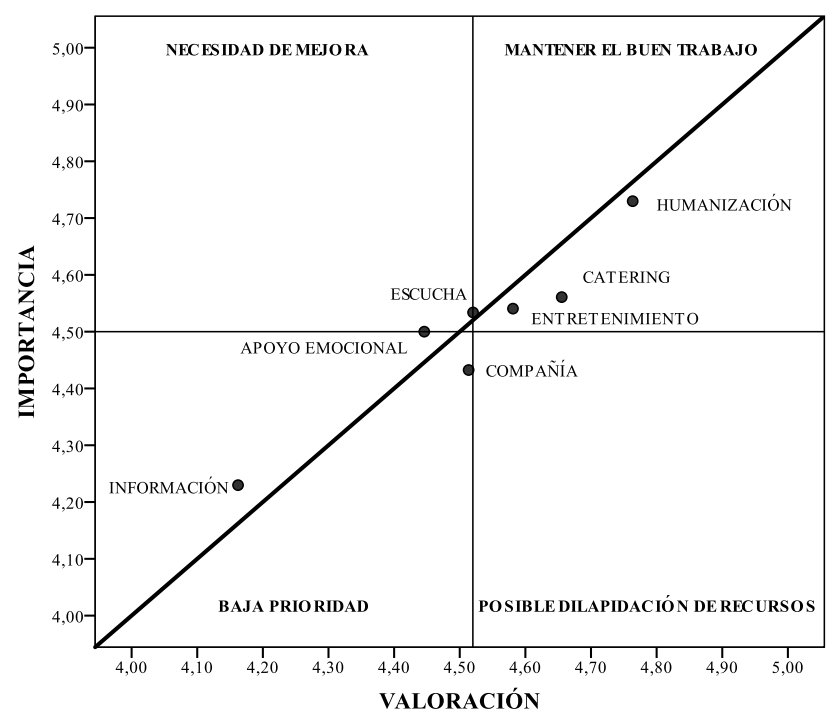

La fiabilidad del instrumento IPA medida como consistencia interna con el $\alpha$ de Cronbach es de 0,926. Como puede observarse en la tabla 1 el atributo mejor valorado es la percepción general del servicio, seguido por la humanización y el catering. En cuanto a la importancia los atributos más puntuados fueron la humanización, la percepción general del servicio y el catering. Respecto a la diferencia nos muestra que los valores negativos (aquellos atributos que tienen posibilidad de mejora) son la escucha a pacientes y familiares, el apoyo emocional y la información útil. El atributo mejor situado respecto a la diferencia es el catering, seguido por la percepción general del servicio y la compañía, el entretenimiento y finalmente la humanización. Todos los atributos se valoran de media entre la puntuación 4 y 5 ("Bueno" y "Muy Bueno").

Si nos fijamos en el análisis gráfico (ver gráfico 1. IPA de los usuarios), observamos 
que la "escucha" es el atributo en el que hay que centrar los esfuerzos de mejora en el futuro, seguido por el apoyo emocional. En cuanto a la información, aunque es mejorable, se sitúa como un factor de muy baja importancia. El resto de atributos (humanización, catering, entretenimiento y compañía) están en el área de mantener el buen trabajo.

No obstante, realizamos un análisis de pacientes y familiares por separado (ver gráficos 2 y 3), ya que entendíamos

\section{Gráfico 2. IPA de los pacientes $(n=68)$}

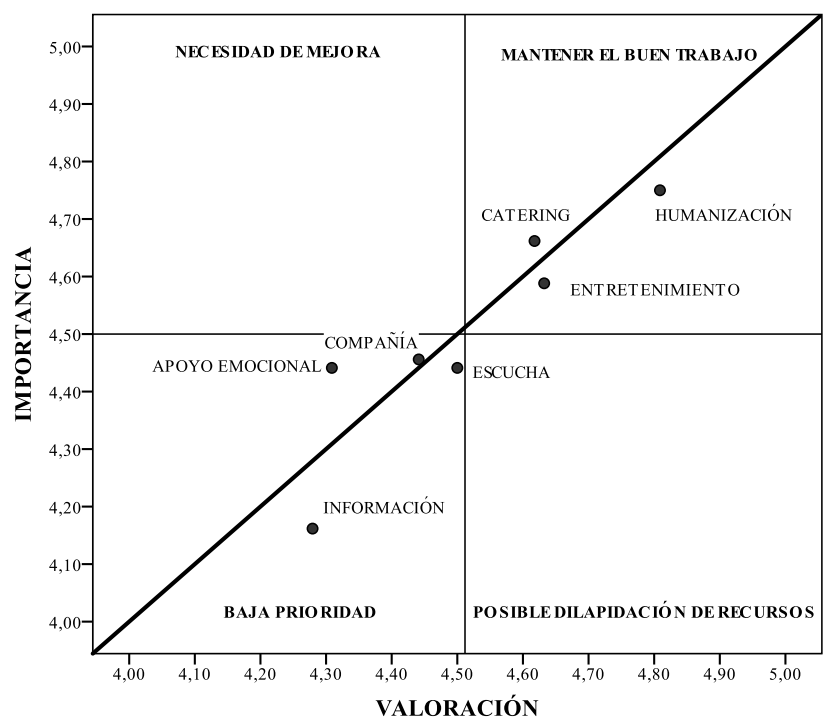

Gráfico 3. IPA de los familiares $(\mathbf{n}=\mathbf{8 0})$

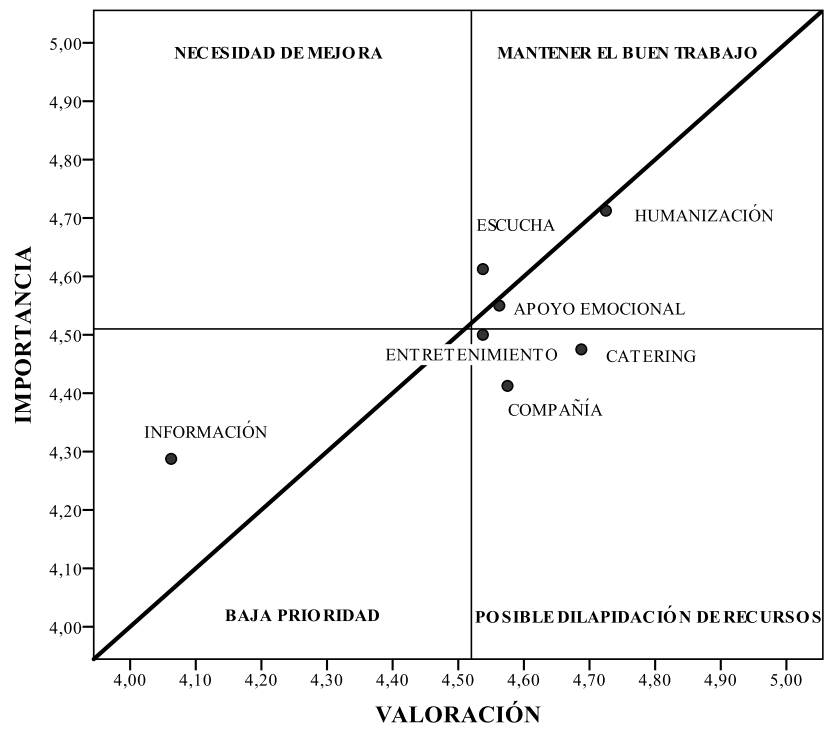


que las necesidades de ambas poblaciones son diferentes. Los pacientes tienen una edad media de 56,31 \pm DT=12,06 años, un $33,8 \%$ de pacientes son hombres. El $46,6 \%$ de pacientes informan de un diagnóstico de cáncer de mama, un 8,6\% fueron diagnosticados de cáncer de próstata y un $6,9 \%$ de cáncer de pulmón. Los familiares tienen una edad de 50,5 $\pm \mathrm{DT}=15,27$ años de media, el $35 \%$ son hombres y el $25,0 \%$ de familiares acompañan a un paciente diagnosticado de cáncer de mama, y el 22,2\% a uno con cáncer de pulmón y un 13,9\% a uno con cáncer de próstata.

Los resultados muestran una satisfacción general similar entre familiares y pacientes, pero la percepción de calidad por atributos muestra diferencias importantes.

Los pacientes perciben posibilidad de mejora en el catering, la compañía y el apoyo emocional. En cuanto a la escucha y la información caen en el área de "baja prioridad". Finalmente, el entretenimiento y la humanización se sitúan en el área de "mantener el buen trabajo". Ver Gráfico 2. IPA de los pacientes.

Los familiares perciben que debe mejorar la "escucha" y la "información", siendo este último atributo de muy baja prioridad. En el caso del entretenimiento, la compañía y el catering, las puntuaciones se sitúan en el área de "posible dilapidación de recursos". Finalmente, la humanización y el apoyo emocional se sitúan en el área de "mantener el buen trabajo". Ver gráfico 3. IPA de los familiares.

El análisis de comparación de medias entre los pacientes y familiares muestran diferencias significativas en la diferencia entre valoración e importancia en el atributo de catering ( $\mathrm{t}=-2,38$; g.l. $=146$; $p<0,05)$, y en el de información $(t=2,16$; g.I.=146; $p<0,05)$. Además, aparece una tendencia en la diferencia valoración-importancia del atributo de compañía $(t=-$ 1,68; g.I.=146; $p<0,05$ a una cola).

\section{DISCUSIÓN}

Si bien el muestreo puede mejorarse en número y perfil de usuario hemos deducido del análisis una aceptable evaluación de la calidad del servicio de catering dirigido a la humanización del entorno de espera hospitalaria y una metodología que permite medir la satisfacción de los usuarios con cada atributo definido del servicio. Además, la metodología ha servido para encontrar que la satisfacción en este servicio se ve influida por el tipo de usuario, por un lado, los pacientes, y por el otro, los acompañantes familiares.

El servicio se percibe con una alta satisfacción general, todas las puntuaciones están en los valores de "bueno" o "muy bueno". Por otro lado, de los siete atributos medidos en este estudio, cuatro de ellos muestran que la diferencia entre importancia y valoración es positiva, lo que significa que el valor del atributo se percibe por encima de las expectativas del usuario del servicio. Estos atributos percibidos como bien valorados son "proporcionar compañía", la "facilitación del entretenimiento", la "humanización" del entorno hospitalario y el "catering". Además, encontramos que los atributos que aún son mejorables son la "escucha a pacientes y familiares", "proporcionar apoyo emocional" y "ofrecer información útil". No obstante, según el análisis gráfico los atributos de información y apoyo emocional pueden acabar siendo de baja prioridad, debido a que son atributos por debajo de la media de importancia.

Cuando diferenciamos entre pacientes y acompañantes familiares encontramos que los resultados muestran una satisfacción global entre "buena" y "muy buena". Las diferencias entre estos dos tipos de usuarios se encuentran en que los atributos mejorables para pacientes son el "catering", "proporcionar compañía" y "proporcionar apoyo emocional". 
Sin embargo, los acompañantes perciben como mejorables los atributos "escucha a pacientes y familiares" y "ofrecer información útil". Por lo que se observa, ambos grupos tienen necesidades diferentes que se expresan en demandas de mejora en áreas del servicio diferentes. Mientras que para los pacientes las demandas se centran en la necesidad de un acompañante que proporcione compañía y soporte emocional, unido a un catering de calidad para entretener la espera, los acompañantes muestran preferencia por la calidad de la escucha y de la información recibida. La escucha y la información pueden considerarse dos atributos que pueden aparecer secuencialmente, por lo tanto, parece razonable que ambos se muestren juntos en esta categoría. En el caso de la información, creemos que probablemente los acompañantes se refieren más a los temas relacionados con los servicios de radioterapia, cuestiones que no entran dentro de las atribuciones del servicio de voluntariado. Interpretamos que este atributo informativo no discrimina entre la información del propio servicio de voluntariado y la información general del servicio de oncología radioterápica. Sería recomendable en un futuro desglosar este atributo en dos para poder aclarar mejor si la procedencia de la información demandada es general o específica del servicio de voluntariado. Otra consecuencia es que posiblemente habría que incluir la evaluación de otros agentes implicados en el servicio como pueden ser el personal sanitario del servicio oncológico que observa todos los días la evolución del voluntariado y de los pacientes.

Como puede deducirse de lo anterior, la diferenciación por tipologías de usuarios permite detectar atributos que se perciben como mejorables que no aparecen en el análisis total, como por ejemplo puede ser el catering. Esto es importante porque existen tipologías de usuarios que pueden tener una diferente sensibilidad a los sabores, o que busquen características parciales del servicio. En un principio, y con el análisis del total de la muestra, estos efectos propios de tipos concretos de usuarios no son posibles de detectar, lo que viene a indicar que una mejora que sugieren nuestros datos de la metodología IPA pasa por definir las clases de usuarios que, por sus características o importancia, deben ser atendidos de manera única y/o especial. En nuestro caso, los pacientes buscan un servicio diferente a los acompañantes/familiares, y esto es necesario conocerlo para poder mejorar el servicio de manera efectiva, siendo el objetivo final contribuir a favorecer la calidad de vida del paciente al ofrecer apoyo y su posible mejora sobre el estado emocional (28). En otros trabajos que usen la técnica IPA en adelante, se deberían caracterizar a los usuarios por perfiles motivacionales que permitan personalizar lo máximo posible el servicio o producto ofrecido.

En definitiva, destacamos que la técnica IPA muestra una alta consistencia interna, unido a que es capaz de discriminar las diferentes áreas de mejora y que, desde una perspectiva global, aparece como una técnica sencilla y fácilmente replicable. Todo ello lleva a concluir que su uso es recomendable para los gestores sanitarios y sociales como medida de calidad y eficacia en el entorno del trabajo de voluntariado sanitario oncológico. Como herramienta se recomienda la aplicación en varios momentos para poder hacer un análisis de evolución del servicio y de las variaciones de percepción en los atributos tras la inclusión de mejoras o cambios en los servicios evaluados. Finalmente, es recomendable que se estudie la influencia de diferentes perfiles de usuarios para poder ofrecer mejoras en los servicios adaptadas a las necesidades del mayor número de usuarios. 


\section{REFERENCIAS BIBLIOGRÁFICAS}

1. Claxton-Oldfield, S. Hospice palliative care volunteers: a review of commonly encountered stressors, how they cope with them, and implications for volunteer training/management. Am J Hosp Palliat Care 2016; 33:201-4. Doi: 10. 10.1177/1049909115571545

2. Woitha, K, Hasselaar, J, van Beek, K, Radbruch, L, Jaspers, B, Engels, Y, Vissers, K. Volunteers in Palliative Care - A Comparison of Seven European Countries: A Descriptive Study. Pain Pract 2014; 28. Doi: 10.1111/papr.12209.

3. Claxton-Oldfield, S, Gosselin, N. How can I help you? A study of the perceived importance of different kinds of hospice palliative care volunteer support. Am J Hosp Palliat Care 2011; 28:271-5. Doi: 10.1177/1049909110385540.

4. Claxton-Oldfield, S, Claxton-Oldfield J, Paulovic S, Wasylkiw L. A study of the motivations of British hospice volunteers. Am J Hosp Palliat Care 2013;30:579-86. Doi: 10.1177/1049909112462057.

5. Claxton-Oldfield S, Claxton-Oldfield J, Paulovic S. Personality traits of British hospice volunteers. Am J Hosp Palliat Care 2013; 30:690-5. Doi: 10.1177/1049909112463117.

6. Claxton-Oldfield S. Hospice palliative care volunteers: The benefits for patients, family caregivers, and the volunteers. Palliat Support Care 2014; 5:1-5. Doi: 10.1017/ S1478951514000674.

7. Garbay M, Gay MC, Claxton-Oldfield S. Motivations, death anxiety, and empathy in hospice volunteers in France. Am J Hosp Palliat Care 2015;32:521-7. Doi: 10.1177/1049909114536978

8. Bender JL, O'Grady L, Jadad AR. Supporting cancer patients through the continuum of care: a view from the age of social networks and computer-mediated communication. Curr Oncol 2008; 15 Sup 2: s107.es42-7.
9. Nissim R, Wong R, Fyles A, Moddel D, Zimmermann C, Rodin G. Can trained volunteers provide psychosocial support to patients undergoing radiotherapy? The perspective of patients and volunteers. Pract Radiat Oncol 2012;2:23-9. Doi: 10.1016/j.pro.2011.11.006.

10. Mackenzie, LJ, Carey, ML, Sanson-Fisher, RW, D'Este, CA. Psychological distress in cancer patients undergoing radiation therapy treatment. Support Care Cancer 2013:1043-51. Doi: 10.1007/s00520012-1624-3.

11. Flory, N, Lang, EV. Distress in the radiology waiting room. Radiology 2011;260:16673. Doi: 10.1148/radiol.11102211.

12. Dolinsky, AL. Considering the competition in strategy development: an extension of importance-performance analysis. J Health Care Mark 1991:11:31-6.

13. Dolinsky, AL., y Caputo, RK. Adding a competitive dimension to importanceperformance analysis: an application to traditional health care systems. Health Care Mark Q 1991:8:61-79.

14. Hawes, JM., Rao, CP. Using ImportancePerformace Analysis to develop health care marketing strategies. J Health Care Mark 1985;5:19-25.

15. Kennedy, DW., Kennedy, SL. Using importance-performance analysis for evaluating university health services. J Am Coll Health 1987; 36:27-31.

16. Leff, EW. Using Importance-Performance Analysis: Importance-performance analysis is a simple tool which yields easily understood data with many uses. Nurse Manage 1990; 21: 20-2.

17. Whynes DK, Reed G. Importance-performance analysis as a guide for hospitals in improving their provision of services. Health Serv Manage Res 1995; 8:266-77.

18. Yavas, U, Shemwell, DJ. Modified importance-performance analysis: an application to hospitals. Int J Health Care Qual Assur Inc Leadersh Health Serv 2001;14:104-10. 
19. Martilla JA., James, JC. Importance-Performance Analysis. J Mark 1977;41:77-9.

20. Oh H. Revisiting importance-performance analysis. Tourism Manage 2001; 22:617-27.

21. Parasuraman A, Zeithaml V, Berry L. A conceptual model of service quality and its implications for future research. J Mark 1985;49:41-50.

22. Abalo Piñeiro, J, Varela Mallou, J, Rial Boubeta, A. El análisis de importanciavaloración aplicado a la gestión de servicios. Psicothema 2006;18:730-7.

23. Abalo Piñeiro, J, Varela Mallou, J, Manzano, V. Importance values for Importance-Performance Analysis: A formula for spreading out values derived for preference rankings. J Bus Res 2007;60:11521. Doi:10.1016/j.jbusres.2006.10.009

24. Bacon DR. A comparison of approaches to Importance-Performance Analy- sis. Inter J Market Res 2003;45: 55-71. Doi:10.4236/jssm.2010.32027

25. Grönroos, C. A Service Quality Model and its marketing implications. Eur J Mark 1984;18:36-44.

26. Parasuraman A, Zeithaml V, Berry L. SERVQUAL: A multiple-item scale for measuring consumer perceptions of service quality. J Retailing 1988;64:12-40.

27. Sampson SE, Showalter MJ. The performance-importance response function: Observations and implications. Serv Ind J 1999;19:1-26 Doi:10.1080/02642069900000027.

28. Villoria E, Fernández C, Padierna C y González C; La intervención psicológica en pacientes oncológicos: una revisión de la literatura (2000-2014). Psicooncología 2015;12:207-36. Doi: 10.5209/ rev_PSIC.2015.v12.n2-3.51005 


\section{ANEXO 1: \\ ENTREVISTA IPA (IMPORTANCE PERFORMANCE ANALYSIS)}
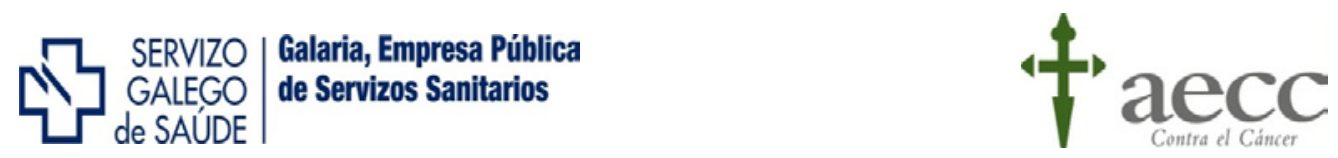

FECHA

No CUESTIONARIO

\section{ENTREVISTA DE CALIDAD “CARRITO DON AMABLE” - PACIENTES / FAMILIARES}

El 16 de enero de 2012 se pone en marcha el programa "Carrito Don Amable" en la Unidad de Oncología Radioterápica del Hospital Meixoeiro. Tras casi un año de desarrollo, hoy solicitamos su colaboración para cumplimentar el presente cuestionario de satisfacción, elaborado con el objetivo de conocer su opinión sobre el mismo y así mejorar la atención a usuarios como usted en el futuro.

1. Beneficiario/a:

Edad: años

Sexo: Hombre $\square$ Mujer $\square$

Lugar de residencia:

Familiar $\square$ Especifique parentesco:

Paciente $\square$ Diagnóstico: Fecha aproximada (mes y año)

Motivo de espera: Consulta $\square$ Tratamiento $\square$ Pruebas $\square$ Seguimiento $\square$

2. ¿Conoce el servicio del "Carrito Don Amable" de la Asociación Española Contra el Cáncer?

Sí $\square$ No $\square$ (Si la respuesta es negativa: FIN del cuestionario)

3. ¿Se han dirigido alguna vez a usted los voluntarios responsables del "Carrito Don Amable"?

Sí $\square$ No $\square$

Si su respuesta es Sí, valore la ACTITUD del voluntario que se dirige a usted:

Escala: Muy malo 1 Malo 2 Regular 3 Bueno 4 Muy bueno 5

\begin{tabular}{|l|l|l|l|l|}
\hline 1 & 2 & 3 & 4 & 5 \\
\hline & & & & \\
\hline
\end{tabular}

4. ¿Sabía que la utilización del Carrito es gratuito? 
5. ¿Qué importancia considera que tienen los siguientes atributos del servicio de voluntariado de la AECC? Escala: Nada importante 1 Poco importante 2 Importancia media 3 Bastante importante 4 Muy importante 5

\begin{tabular}{|l|l|l|l|l|l|}
\hline \multicolumn{1}{|c|}{ IMPORTANCIA } & $\mathbf{1}$ & $\mathbf{2}$ & $\mathbf{3}$ & $\mathbf{4}$ & $\mathbf{5}$ \\
\hline Proporciona compañía & & & & & \\
\hline Escucha a pacientes y familiares & & & & & \\
\hline Proporciona apoyo emocional & & & & & \\
\hline Facilita entretenimiento en la espera & & & & & \\
\hline Contribuye a que el hospital parezca más humano & & & & \\
\hline Ofrece información útil & & & & & \\
\hline Catering & & & & & \\
\hline Importancia general del servicio & & & & & \\
\hline
\end{tabular}

6. ¿Añadiría algún producto al servicio de CATERING?

No $\square$ Sí $\square$ Especifique cuál/es:

7. ¿Le genera algún tipo de problema o incomodidad la presencia del "Carrito Don Amable" No $\square$ Sí

Especifique en qué consiste:

8. ¿Qué valoración considera que tienen los siguientes atributos del servicio de voluntariado de la AECC? Escala: Muy malo 1 Malo 2 Regular 3 Bueno 4 Muy bueno 5

\begin{tabular}{|l|l|l|l|l|l|}
\hline \multicolumn{1}{|c|}{ VALORACIÓN } & $\mathbf{1}$ & $\mathbf{2}$ & $\mathbf{3}$ & $\mathbf{4}$ & $\mathbf{5}$ \\
\hline Proporciona compañía & & & & & \\
\hline Escucha a pacientes y familiares & & & & & \\
\hline Proporciona apoyo emocional & & & & & \\
\hline Facilita entretenimiento en la espera & & & & & \\
\hline Contribuye a que el hospital parezca más humano & & & & \\
\hline Ofrece información útil & & & & & \\
\hline Catering & & & & & \\
\hline Valoración general del servicio & & & & & \\
\hline
\end{tabular}

9. Valore la presencia de la Asociación Española Contra el Cáncer (tanto el servicio del "Carrito" como los voluntarios/as que lo dirigen) en esta Unidad de Oncología Radioterápica: Escala: Muy malo 1 Malo 2 Regular 3 Bueno 4 Muy bueno 5

\begin{tabular}{|l|l|l|l|l|}
\hline 1 & 2 & 3 & 4 & 5 \\
\hline & & & & \\
\hline
\end{tabular}

10. ¿Desea añadir el siguiente comentario sobre el "Carrito Don Amable"?: 
